\title{
THE EQUIVARIANT SERRE SPECTRAL SEQUENCE
}

\author{
I. MOERDIJK AND J.-A. SVENSSON
}

(Communicated by Frederick R. Cohen)

\begin{abstract}
For spaces with a group action, we introduce Bredon cohomology with local (or twisted) coefficients and show that it is invariant under weak equivariant homotopy equivalence. We use this new cohomology to construct a Serre spectral sequence for equivariant fibrations.
\end{abstract}

Bredon [1] introduced what is now called Bredon cohomology, with the purpose of developing obstruction theory in the context of spaces equipped with an action of a fixed group $G$. The kind of coefficients needed in this theory are not abelian groups but rather contravariant functors from the orbit category $\mathscr{O}(G)$ into abelian groups.

The purpose of this paper is to prove the existence of a spectral sequence for a Serre fibration of $G$-spaces. As in the nonequivariant case, if no further restrictions are made, it is necessary to use cohomology with twisted coefficients. Such twisted coefficients are not functors on the orbit category $\mathscr{O}(G)$, but on some augmentation of $\mathscr{O}(G)$ depending on the base space of the fibration.

Our approach is to give a new definition of Bredon cohomology in terms of cohomology of categories. Indeed, with a $G$-space $X$ we shall associate a category $\Delta_{G}(X)$ of "equivariant singular simplices in $X$." Any abelian groupvalued functor $M$ from the orbit category $\mathscr{O}(G)$ can be viewed as a system of coefficients on the category $\Delta_{G}(X)$. A key result is that for any such $M$, the cohomology groups $H^{*}\left(\Delta_{G} X, M\right)$ of this category are naturally isomorphic to the Bredon cohomology groups $H_{G}^{*}(X, M)$; see Theorem 2.2.

The category $\Delta_{G}(X)$ allows us to define cohomology groups of a $G$-space with more general coefficients. In particular, we shall construct a category $\Pi_{G}(X)$ of "equivariant homotopy classes of paths in $X$," which sits in between $\Delta_{G}(X)$ and $\mathscr{O}(G)$ by functors $\Delta_{G}(X) \rightarrow \Pi_{G}(X) \rightarrow \mathscr{O}(G)$. A twisted or local system of coefficients $M$ on $X$ is then defined as an abelian group-valued functor on $\Pi_{G}(X)$, and the Bredon cohomology of $X$ with twisted coefficients can now be constructed as the cohomology $H^{*}\left(\Delta_{G}(X), M\right)$ of the category $\Delta_{G}(X)$. This is invariant under weak $G$-homotopy equivalence, see Theorem 2.3. (The converse is also true: a map of $G$-spaces is a weak $G$-homotopy equivalence whenever it induces an equivalence of fundamental groupoids as well as an

Received by the editors September 21, 1990 and, in revised form, August 19, 1991.

1991 Mathematics Subject Classification. Primary 55N91, 55R91, 55T91.

The authors gratefully acknowledge financial support from the Swedish Natural Science Research Council and the Netherlands Science Organisation (NWO). 
isomorphism in cohomology with such twisted coefficients; see MoerdijkSvensson [8].) This notion of local coefficients should not be confused with a different one introduced in Bredon [1]. For example, unlike Bredon's notion, ours reduces in the nonequivariant context to the usual one originating with Steenrod.

A Serre fibration $f: Y \rightarrow X$ of $G$-spaces and a (twisted) coefficient system $M$ on $Y$ then naturally give rise to a twisted coefficient system $H^{*}(f, M)$ on $X$, which should be thought of as the Bredon cohomology of the fibers of $f$ (see 3.1). The Serre spectral sequence now takes the usual form $H_{G}^{p}\left(X, H_{G}^{q}(f, M)\right)$ $\Rightarrow H_{G}^{p+q}(Y, M)$.

We point out that analogous results for Bredon homology can be derived in the same way. The interested reader will also notice that our approach can be carried out for arbitrary diagrams of spaces. For example, this gives a Serre spectral sequence for cyclic (co-)homology.

\section{COHOMOLOGY OF CATEGORIES}

In this section, we review the definition and some well-known basic facts concerning cohomology groups of categories (cf., e.g., Quillen [9], Gabriel-Zisman [5, Appendix II], and Watts [13]).

Let $\mathbb{C}$ be a small category. We write $\underline{A b}(\mathbb{C})$ for the category with objects the contravariant functors from $\mathbb{C}$ into abelian groups and with morphisms the natural transformations. $\underline{\mathrm{Ab}}=\underline{\mathrm{Ab}}(1)$ is the category of abelian groups. There is a 'global sections'-or $\varlimsup_{-}$-functor

$$
\Gamma=\Gamma_{\mathbb{C}}: \underline{\mathrm{Ab}}(\mathbb{C}) \rightarrow \underline{\mathrm{Ab}}
$$

defined by $\Gamma(M)=\lim M=\left\{\alpha: \mathbb{C}_{0} \rightarrow M \mid M(u)(\alpha(C))=\alpha(D)\right.$ for every morphism $u: D \rightarrow C$ in $\mathbb{C}\}$. (Here $\mathbb{C}_{0}$ is the set of objects of $\mathbb{C}$.) $A b(\mathbb{C})$ has enough injectives, so one can form the right derived functors of $\Gamma$. These are, by definition, the cohomology groups of $\mathbb{C}$ :

$$
H^{n}(\mathbb{C}, M)=R^{n} \Gamma(M), \quad n \geq 0 .
$$

For a small category $\mathbb{C}$, let $N \mathbb{C}$ denote the nerve of $\mathbb{C}$, i.e., the simplicial set with $n$-simplices the composable sequences $u=\left(C_{0} \rightarrow^{u_{1}} C_{1} \rightarrow u_{2} \ldots \rightarrow{ }^{u_{n}} C_{n}\right)$ in $\mathbb{C}$. If $M \in \underline{\mathrm{Ab}}(\mathbb{C})$, one can form a cosimplicial abelian group $C^{\bullet}(\mathbb{C}, M)$ defined by

$$
C^{n}(\mathbb{C}, M)=\prod_{u \in N_{n}(\mathbb{C})} M(u(0))
$$

(Here, as in the rest of this paper, if $u=\left(C_{0} \rightarrow^{u_{1}} C_{1} \rightarrow \cdots \rightarrow^{u_{n}} C_{n}\right)$ is an element of $N_{n}(\mathbb{C})$, we write $u(i)=C_{i}$.) The differentials in the associated cochain complex (also denoted by $C^{\bullet}(\mathbb{C}, M)$ ) are obtained from the face maps of $N \mathbb{C}$ by taking alternating sums, in the standard way. This cochain complex computes the cohomology of $\mathbb{C}$ with coefficients in $M:$ there is a canonical isomorphism

$$
H^{*}(\mathbb{C}, M) \cong H^{*}\left(C^{\bullet}(\mathbb{C}, M)\right)
$$

For later reference, we single out the special case where $\mathbb{C}$ is the dual $\Delta^{\text {op }}$ of the simplicial category $\Delta$. Then $\underline{A b}\left(\Delta^{\text {op }}\right)$ is the category of cosimplicial abelian groups. For a cosimplicial group $M$, the cohomology $H^{*}\left(\Delta^{\text {op }}, M\right)$ can 
be computed as the cohomology of the cochain complex $M^{0} \rightarrow M^{1} \rightarrow M^{2} \rightarrow \cdots$ obtained from $M$ by taking alternating sums of the coface maps $M^{n} \rightarrow M^{n+1}$; briefly,

$$
H^{*}\left(\Delta^{\mathrm{op}}, M\right) \cong H^{*}\left(M^{\bullet}\right) .
$$

For an arbitrary small category $\mathbb{C}$, an $M \in \underline{\mathrm{Ab}}(\mathbb{C})$ is called locally constant, or morphism inverting, if for any arrow $u: D \rightarrow C$ in $\mathbb{C}, M$ gives an isomorphism $M(u): M(C) \stackrel{\sim}{\rightarrow} M(D)$ of abelian groups. A locally constant $M$ induces a locally constant (or twisted) system of coefficients on the classifying space $B \mathbb{C}$ of $\mathbb{C}$, again denoted by $M$. Now $H^{*}(\mathbb{C}, M)$ coincides with the usual twisted cohomology of the space $B \mathbb{C}$ :

$$
H^{*}(\mathbb{C}, M) \cong H^{n}(B \mathbb{C}, M) \quad(\text { for } M \text { locally constant }) .
$$

To conclude this section, we recall the Grothendieck spectral sequence (Grothendieck [7]) in the context of cohomology of small categories. Let $f: \mathbb{D}$ $\rightarrow \mathbb{C}$ be a functor between small categories. There are induced functors

$$
\underline{\mathrm{Ab}}(\mathbb{D}) \underset{f^{*}}{\stackrel{f_{*}}{\rightleftarrows}} \underline{\mathrm{Ab}}(\mathbb{C})
$$

defined by

$$
\begin{aligned}
f^{*}(M)(D) & =M(f D) & & (D \in \mathbb{D}), \\
f_{*}(N)(C) & =\overleftarrow{l i m}_{f / C}^{\operatorname{li}} N & & (C \in \mathbb{C}),
\end{aligned}
$$

where $f / C$ is the comma category. (Objects of $f / C$ are pairs $(D, u: f D \rightarrow$ $C)$ and morphisms from $(D, u: f D \rightarrow C)$ to $\left(D^{\prime}, u^{\prime}: f D^{\prime} \rightarrow C\right)$ are maps $\alpha: D \rightarrow D^{\prime}$ such that $u^{\prime} f(\alpha)=u$.) The functor $f^{*}$ induces a homomorphism

$$
f^{*}: H^{n}(\mathbb{C}, M) \rightarrow H^{n}\left(\mathbb{D}, f^{*} M\right) .
$$

If $f: \mathbb{D} \rightarrow \mathbb{C}$ is a weak equivalence (in the sense that $B f: B \mathbb{D} \rightarrow B \mathbb{C}$ is a homotopy equivalence of spaces), then the map (8) is an isomorphism, provided $M$ is locally constant.

If $N \in \underline{\mathrm{Ab}}(\mathbb{D})$ then there is a canonical isomorphism $\Gamma_{\mathbb{C}} f_{*} N \cong \Gamma_{\mathbb{D}} N$, and hence a Grothendieck spectral sequence (natural in $f$ )

$$
E_{2}^{p, q}=R^{p} \Gamma_{\mathbb{C}}\left(R^{q} f_{*}(N)\right) \Rightarrow R^{p+q} \Gamma_{\mathbb{D}}(N) .
$$

By the explicit description of $f_{*}$ given above, this spectral sequence may also be written as

$$
E_{2}^{p, q}=H^{p}\left(\mathbb{C}, H^{q}(f /-, N)\right) \Rightarrow H^{p+q}(\mathbb{D}, N) .
$$

Here $H^{q}(f /-, N) \in \underline{\mathrm{Ab}}(\mathbb{C})$ is the functor that sends an object $C$ of $\mathbb{C}$ to $H^{q}\left(f / C, \omega_{C}^{*}(N)\right)$, where $\omega_{C}: f / C \rightarrow \mathbb{D}$ is the forgetful functor $(D, u) \mapsto D$.

\section{BREDON COHOMOLOGY WITH TWISTED COEFFICIENTS}

Let $G$ be a (discrete) group and $X$ a $G$-space. The purpose of this section is to express (ordinary) Bredon cohomology of $X$ as the cohomology of a certain category $\Delta_{G}(X)$ and to introduce twisted Bredon cohomology. In $\S 3$, we derive a Serre spectral sequence for a $G$-fibration $f: Y \rightarrow X$, from the Grothendieck spectral sequence of the functor $\Delta_{G}(Y) \rightarrow \Delta_{G}(X)$. 
The construction of $\Delta_{G}(X)$ proceeds by the so-called Grothendieck construction (see, e.g., Gray [6] and Thomason [12]). If $\mathbb{C}$ is a small category and $F: \mathbb{C}^{\text {op }} \rightarrow$ Cat is a functor into the category Cat of small categories, then the Grothendieck construction of $F$ is the category $\int_{\mathbb{C}} F$ defined as follows: the objects of $\int_{\mathbb{C}} F$ are pairs $(C, x)$ where $C$ is an object of $\mathbb{C}$ and $x$ one of $F(C)$; an arrow $(C, x) \rightarrow\left(C^{\prime}, x^{\prime}\right)$ in $\int_{\mathbb{C}} F$ is a pair $(f, \alpha)$ where $f: C \rightarrow C^{\prime}$ in $\mathbb{C}$ and $\alpha: x \rightarrow F(f)\left(x^{\prime}\right)$ in $F(C)$; composition in $\int_{\mathbb{C}} F$ is defined in the evident way. Notice that there is a canonical projection functor

$$
\int_{\mathbb{C}} F \rightarrow \mathbb{C}
$$

The Grothendieck construction is natural in $\mathbb{C}$ and $F$ in the obvious sense.

Thomason [12] showed that the Grothendieck construction provides a categorical model for homotopy colimits, in the sense that for a functor $F: \mathbb{C}^{\text {op }} \rightarrow$ Cat there is a (weak) homotopy equivalence

$$
\underset{\mathbb{C}^{\mathrm{op}}}{\operatorname{hocolim}} B \circ F \simeq B\left(\int_{\mathbb{C}} F\right),
$$

where $B: \underline{\text { Cat }} \rightarrow$ Top is the classifying space functor.

For example, if $T$ is a topological space with associated singular complex $S_{\bullet}(T): \Delta^{\text {op }} \rightarrow \underline{\text { Sets }} \subseteq \underline{\text { Cat }}$, write $\Delta(T)=\int_{\Delta} S_{\bullet}(T)$. The category $\Delta(T)$ faithfully represents the space $T$, up to homotopy. Indeed, there are (weak) homotopy equivalences

$$
T \simeq\left|S_{\bullet}(T)\right| \simeq \underset{\Delta^{\mathrm{op}}}{\operatorname{hocolim}} S_{\bullet}(T) \simeq B \Delta(T)=\underset{\Delta(T)^{\mathrm{op}}}{\operatorname{hocolim}} p t ;
$$

here the second homotopy equivalence is standard (see, e.g., [10, Appendix]), the third is a special case of (12), and the last equality holds by definition.

Now consider a fixed group $G$, and write $\mathscr{O}(G)$ for the orbit category; i.e., $\mathscr{O}(G)$ is the full subcategory of the category of $G$-spaces spanned by the (discrete) $G$-spaces $G / H$, where $H$ is any subgroup of $G$. For a $G$-space $X$, there is a functor

$$
X^{(-)}: \mathscr{O}(G)^{\mathrm{op}} \rightarrow \underline{\text { Top }}
$$

into the category Top of topological spaces, which sends an object $G / H$ of $\mathscr{O}(G)$ to the space $\operatorname{Map}_{G}(G / H, X)=X^{H}$ of points fixed by $H$. Composing

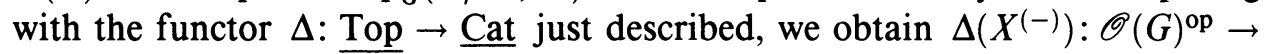
Cat. Let

$$
\Delta_{G}(X)=\int_{\mathscr{O}(G)} \Delta\left(X^{(-)}\right),
$$

with projection functor $p_{X}: \Delta_{G}(X) \rightarrow \mathscr{O}(G)$ as in (11).

The category $\Delta_{G}(X)$ can be described explicitly as follows: its objects are $G$-maps $\sigma: G / H \times \Delta^{n} \rightarrow X$, where $\Delta^{n}$ is the standard $n$-simplex; maps from $\left(\sigma: G / H \times \Delta^{n} \rightarrow X\right)$ to $\left(\tau: G / K \times \Delta^{m} \rightarrow X\right)$ are pairs $(\varphi, \alpha)$ where $\varphi: G / H \rightarrow$ $G / K$ is a $G$-map and $\alpha: \Delta^{n} \rightarrow \Delta^{m}$ is a simplicial operator (i.e., a map coming from an order preserving function $\{0, \ldots, n\} \rightarrow\{0, \ldots, m\})$ such that $\tau$ 。 $(\varphi \times \alpha)=\sigma$. 
The category $\Delta_{G}(X)$ is in some sense the equivariant analogue of the category $\Delta(T)$ associated to a topological space $T$. Thus, analogous to (13), there is a weak $G$-homotopy equivalence

$$
X \simeq \underset{\Delta_{G}(X)}{\operatorname{hocolim}} p_{X}
$$

cf. Seymour [11] and Elmendorf [4]. (Indeed, using Elmendorf's notation, this is his weak equivalence $C \Phi X \stackrel{\sim}{\rightarrow} X$, except that the geometric realization involved in the definition of the functor " $C$ " is replaced by the thick realization of Segal [10, Appendix].)

Another important category associated to a $G$-space $X$ is the category $\Pi_{G}(X)$ constructed as follows. Let $\pi\left(X^{(-)}\right): \mathscr{O}(G)^{\text {op }} \rightarrow$ (groupoids) $\subseteq$ Cat be the functor that sends $G / H$ to the fundamental groupoid $\pi\left(X^{H}\right)$ of the subspace $X^{H}$ of $H$-fixed points. Then define

$$
\pi_{G}(X)=\int_{\mathscr{O}(G)} \pi\left(X^{(-)}\right)
$$

with projection $q_{X}: \pi_{G}(X) \rightarrow \mathscr{O}(G)$ as in (11).

Explicitly, $\pi_{G}(X)$ is the category whose objects are $G$-maps $x: G / H \rightarrow X$ and whose arrows from $(x: G / H \rightarrow X)$ to $(y: G / K \rightarrow X)$ are pairs $(\varphi, \alpha)$ where $\varphi: G / H \rightarrow G / K$ is a $G$-map and $\alpha$ is a $G$-homotopy class (relative endpoints) of equivariant paths $G / H \times I \rightarrow X$ from $x \circ \varphi$ to $y$. We shall call $\pi_{G}(X)$ (as well as the functor $\pi\left(X^{(-)}\right)$) the fundamental groupoid of the $G$ space $X$-but notice that $\pi_{G}(X)$ is not a groupoid, and that in general $\pi_{G}(X) \neq$ $\pi\left(\Delta_{G}(X)\right)$ (unless $G$ is trivial).

There is a canonical functor from $\Delta_{G}(X)$ to $\pi_{G}(X)$, commuting with the projections:

$$
\begin{array}{ccc}
\Delta_{G}(X) & \stackrel{v_{X}}{\rightarrow} \pi_{G}(X) \\
p_{X} \searrow & \swarrow q_{X} \\
\mathscr{O}(G) &
\end{array}
$$

Indeed, if $T$ is any topological space, there is a quotient functor $\Delta(T) \rightarrow \pi(T)$ that sends an object $\left(\sigma: \Delta^{n} \rightarrow T\right)$ to $\sigma\left(e^{n}\right) \in T$, where $e^{n}$ is the last ( $n$ th) vertex of $\Delta^{n}$; an arrow $\alpha:\left(\sigma: \Delta^{n} \rightarrow T\right) \rightarrow\left(\tau: \Delta^{m} \rightarrow T\right)$ of $\Delta(T)$ is sent to the image under $\tau$ of the linear path from $\alpha\left(e^{n}\right)$ to $e^{m}$ in $\Delta^{m}$. This functor is natural in $T$, so for a $G$-space $X$ we obtain a natural transformation $\Delta\left(X^{(-)}\right) \rightarrow \pi\left(X^{(-)}\right)$between functors $\mathscr{O}(G)^{\text {op }} \rightarrow \underline{\text { Cat }}$, and hence by "integration" a functor $v_{X}$ as in (18).

We are now ready to define Bredon cohomology with twisted coefficients. A functor $M \in \underline{\mathrm{Ab}}\left(\Delta_{G}(X)\right)$ is said to be a $(G$-)local (or twisted, or locally constant, relative to $G$ ) system on $X$ if, up to isomorphism, it factors through $v_{X}: \Delta_{G}(X) \rightarrow \pi_{G}(X)$ (i.e., $M \cong v_{X}^{*}\left(M^{\prime}\right)$ for some $\left.M^{\prime} \in \underline{\mathrm{Ab}}\left(\pi_{G}(X)\right)\right)$. Similarly, $M$ is said to be constant (relative to $G$ ) if it factors through $p_{X}: \Delta_{G}(X) \rightarrow$ $\mathscr{O}(G)$, up to isomorphism. Observe that when $X$ is $G$-simply connected (in the sense that $X^{H}$ is nonempty and simply connected for any subgroup $H \subseteq G$ ) then the functor $q_{X}$ is an equivalence of categories, hence every locally constant system is constant.

2.1. Definition. For a $G$-local system $M$ on a $G$-space $X$, the (twisted) Bredon cohomology $H_{G}^{*}(X, M)$ of $X$ with coefficients in $M$ is defined as

$$
H_{G}^{*}(X, M)=H^{*}\left(\Delta_{G}(X), M\right) .
$$


We will use the notation $H_{G}^{*}(X, M)$ only for local coefficients $M$ (although (19) makes sense for any $M \in \underline{\mathrm{Ab}}\left(\Delta_{G}(X)\right)$. In 2.3 we will see that a weak $G$-homotopy equivalence $f: Y \rightarrow X$ induces an isomorphism $H_{G}^{*}(X, M) \stackrel{\sim}{\rightarrow}$ $H_{G}^{*}\left(Y, f^{*} M\right)$; this is not true for general coefficients.

To justify the notation introduced in 2.1 , we will now prove that for a constant system $M$, it agrees with ordinary Bredon cohomology. We briefly recall the singular version of Bredon cohomology from Bröcker [2]. For a $G$-space $X$ and $n \geq 0$, let

$$
C_{n}\left(X^{(-)}\right): \mathscr{O}(G)^{\mathrm{op}} \rightarrow \underline{\mathrm{Ab}}
$$

be the functor that to $G / H$ associates the free abelian group $\mathbb{Z}\left[S_{n}\left(X^{H}\right)\right]$ generated by the singular $n$-simplices of $X^{H}$. For $M \in \underline{\mathrm{Ab}}(\mathscr{O}(G))$, let

$$
C^{n}(X, M)=\operatorname{Hom}_{\mathscr{Q}(G)}\left(C_{n}(X), M\right)
$$

be the abelian group of natural transformations from $C_{n}(X)$ into $M$. Defining the differentials in the standard way, we obtain a cochain complex $C^{\bullet}(X, M)$ and Bröcker's definition of Bredon cohomology is

$$
H_{B r}^{*}(X, M)=H^{*}\left(C^{\bullet}(X, M)\right) \text {. }
$$

2.2. Theorem. Let $X$ be a $G$-space and $M$ a functor $\mathscr{O}(G)^{\mathrm{op}} \rightarrow \underline{\mathrm{Ab}}$. Then there is an isomorphism

$$
H_{B r}^{*}(X, M) \cong H_{G}^{*}(X, M),
$$

natural in $X$ and $M$. (On the right we identify $M$ with $M \circ p_{X}: \Delta_{G}(X)^{\mathrm{op}} \rightarrow$ Ab.)

Proof. Consider the bisimplicial set $\widetilde{X}$ whose $p, q$-simplices are triples $(u, \alpha$, $\sigma)$, where $u=\left(\Delta^{n_{0}} \rightarrow^{u_{1}} \Delta^{n_{1}} \rightarrow \cdots \rightarrow \rightarrow^{u_{p}} \Delta^{n_{p}}\right) \in N_{p}(\Delta)$ and $\alpha=\left(G / H_{0} \rightarrow^{\alpha_{1}}\right.$ $\left.G / H_{1} \rightarrow \cdots \rightarrow^{\alpha_{q}} G / H_{q}\right) \in N_{q}(\mathscr{O}(G))$ while $\sigma: G / H_{q} \times \Delta^{n_{p}} \rightarrow X$ is a $G$-map. Observe that

$$
\operatorname{diagonal}(\widetilde{X}) \cong N\left(\Delta_{G} X\right) \text {. }
$$

From $\tilde{X}$ and the given functor $M: \mathscr{O}(G)^{\mathrm{op}} \rightarrow \underline{\mathrm{Ab}}$ we obtain a double cosimplicial abelian group $C^{\bullet \bullet}(X, M)$ in the obvious way,

$$
C^{p, q}(X, M)=\prod_{(u, \alpha, \sigma) \in \widetilde{X}^{p . q}} M(\alpha(0))
$$

(recall $\alpha(0)=G / H_{0}$ for $\alpha$ as above). We also write $C^{\bullet \bullet}(X, M)$ for the associated double cochain complex, and $\operatorname{Tot}\left(C^{\bullet \bullet}(X, M)\right)$ for the associated total complex. Then by a result of Dold and Puppe [3], by (23) and (3) we obtain

$$
\begin{aligned}
H^{n}\left(\operatorname{Tot} C^{\bullet \bullet}(X, M)\right) & \cong H^{n}\left(\operatorname{diag} C^{\bullet \bullet}(X, M)\right) \\
& \cong H^{n}\left(\Delta_{G}(X), p_{X}^{*} M\right)=H_{G}^{n}(X, M),
\end{aligned}
$$

where the last equality holds by definition (we identify $M$ with $p_{X}^{*}(M)$ ).

Now let us consider the rows of $\widetilde{X}$ and of the complex $C^{\bullet \bullet}(X, M)$ separately. For $(u, \alpha, \sigma) \in \tilde{X}^{p, q}$ as above, we may identify $(\alpha, \sigma)$ with a $q$ simplex of the nerve of $\int_{\mathscr{Q}(G)} S_{n_{p}}\left(X^{(-)}\right)$. Thus for fixed $p \geq 0$, the complex 
$C^{p, \bullet}(X, M)$ is a product of complexes computing the cohomology of the categories $\int_{\mathcal{O}(G)} S_{n_{p}}\left(X^{(-)}\right)$with coefficients in $M$. Therefore, still keeping $p$ fixed,

$$
H^{q}\left(C^{p, \bullet}(X, M)\right)=\prod_{u \in N_{p}(\Delta)} H^{q}\left(\int_{\mathscr{O}(G)} S_{n_{p}}\left(X^{(-)}\right), M\right)
$$

(here $n_{p}$ depends on $u=\left(\Delta^{n_{0}} \rightarrow \cdots \rightarrow \Delta^{n_{p}}\right)$, as above). We shall prove below that for any fixed $n \geq 0$

$$
H^{q}\left(\int_{\mathscr{O}(G)} S_{n}\left(X^{(-)}\right), M\right)=\left\{\begin{array}{l}
0 \text { if } q>0, \\
\operatorname{Hom}_{\mathscr{O}(G)}\left(C_{n}(X), M\right) \text { if } q=0 .
\end{array}\right.
$$

The theorem then follows, since by (26), (27), and (5) we have

$$
\begin{aligned}
H^{p}\left(\operatorname{Tot} C^{\bullet \bullet}(X, M)\right) & \cong H^{p}\left(\prod_{u \in N(\Delta)} \operatorname{Hom}_{\mathscr{Q}(G)}\left(C_{n}(X), M\right)\right) \\
& \cong H^{p}\left(\Delta^{\text {op }}, \operatorname{Hom}_{\mathscr{Q}(G)}\left(C_{\bullet}(X), M\right)\right) \\
& \cong H^{p}\left(\operatorname{Hom}_{\mathscr{O}(G)}\left(C_{\bullet}(X), M\right)\right) \\
& =H_{B r}^{p}(X, M) \quad(\text { by definition }) .
\end{aligned}
$$

This isomorphism together with (25) gives $H_{G}^{p}(X, M) \cong H_{B r}^{p}(X, M)$, as required.

It remains to show (27). Let $\operatorname{Map}\left(\Delta^{n}, X\right)$ be the $G$-set of maps $\Delta^{n} \rightarrow X$ with $G$-action induced from $X$. Then

$$
C_{n}(X)(G / H)=\mathbb{Z}\left[S_{n}\left(X^{H}\right)\right]=\mathbb{Z}\left[\operatorname{Map}\left(\Delta^{n}, X\right)^{H}\right] .
$$

Therefore to prove (27), it suffices to show that for an arbitrary $G$-set $S$,

$$
H^{q}\left(\int_{\mathscr{Q}(G)} S^{(-)}, M\right)=\left\{\begin{array}{l}
0, \quad q>0, \\
\operatorname{Hom}_{\mathscr{O}(G)}\left(\mathbb{Z}\left[S^{(-)}\right], M\right), \quad q=0 .
\end{array}\right.
$$

By writing $S$ as a sum of orbits, it suffices to consider the case where $S=G / H$ for a fixed subgroup $H \subseteq G$. But in this case we have

$$
\int_{\mathscr{O}(G)}\left(S^{(-)}\right) \cong \mathscr{O}(G) /(G / H)
$$

and $\Gamma: \underline{\mathrm{Ab}}\left(\int_{\mathscr{O}(G)}\left(S^{(-)}\right)\right) \rightarrow \underline{\mathrm{Ab}}$ is simply the evaluation at $(G / H$, id: $G / H \rightarrow$ $G / H)$, which is an exact functor. Thus writing $p: \int_{\mathscr{O}(G)}\left(S^{(-)}\right) \rightarrow \mathscr{O}(G)$ for the projection functor, we have for $M \in \underline{\mathrm{Ab}}(\mathscr{O}(G))$ that $H^{q}\left(\int_{\mathscr{O}(G)}\left(S^{(-)}\right), p^{*} M\right)=0$ if $q>0$ and $H^{0}\left(\int_{\mathscr{O}(G)}\left(S^{(-)}\right), p^{*} M\right)=p^{*}(M)((G / H)$, id $)=M(G / H)$ for $q=0$ (and $S=G / H)$. This proves (28), and completes the proof of the theorem.

We now deduce the invariance under weak equivalence. Recall that a $G$-map $f: Y \rightarrow X$ of $G$-spaces is a weak $G$-homotopy equivalence if for any subgroup $H \subseteq G, f$ restricts to an ordinary weak homotopy equivalence $f^{H}: Y^{H} \rightarrow X^{H}$. In the following theorem, we denote by $f^{*} M$ the local system on $Y$ induced by a local system $M$ on $X$ (i.e., $f^{*} M$ is the composition $\Delta_{G}(Y)^{\text {op }} \rightarrow^{\Delta_{G}(f)}$ $\left.\Delta_{G}(X)^{\mathrm{op}} \rightarrow^{M} \underline{\mathrm{Ab}}\right)$. 
2.3. Theorem. Let $f: Y \rightarrow X$ be a weak G-homotopy equivalence. Then for any local system $M$ on $X$, the map $f$ induces an isomorphism $H_{G}^{*}(X, M) \stackrel{\sim}{\rightarrow}$ $H_{G}^{*}\left(Y, f^{*} M\right)$.

Proof. Suppose given, a system $M: \Delta_{G}(X)^{\mathrm{op}} \rightarrow \underline{\mathrm{Ab}}$ that factors through $v_{X}$ : $\Delta_{G}(X) \rightarrow \Pi_{G}(X)$ (up to isomorphism). As in the proof of 2.2, we can construct a bisimplicial set $\widetilde{X}$ and a bicomplex $C^{\bullet \bullet}(X, M)$, where

$$
C^{p, q}(X, M)=\prod_{(u, \alpha, \sigma) \in \widetilde{X}^{p, q}} M(\sigma(\alpha \times u)) .
$$

Here for $u=\left(\Delta^{n_{0}} \rightarrow^{u_{1}} \cdots \rightarrow^{u_{p}} \Delta^{n_{p}}\right), \alpha=\left(G / H_{0} \rightarrow^{\alpha_{1}} \cdots \rightarrow^{\alpha_{q}} G / H_{q}\right)$, and $\sigma: G / H_{q} \times \Delta^{n_{p}} \rightarrow X$ as before, $\sigma(\alpha \times u)$ denotes the obvious composition $\left(G / H_{0} \times \Delta^{n_{0}} \rightarrow X\right) \in \Delta_{G}(X)$. As in the proof of 2.2, we have

$$
H^{n}\left(\operatorname{Tot} C^{\bullet \bullet}(X, M)\right) \cong H_{G}^{n}(X, M) \text {. }
$$

Also as in 2.2 , the rows of the double complex $C(X, M)$ are exact, so one of the spectral sequences associated to (29) degenerates. For the other spectral sequence, we introduce the following notation. For $\alpha=\left(G / H_{0} \rightarrow^{\alpha_{1}} \ldots \rightarrow^{\alpha_{q}}\right.$ $\left.G / H_{q}\right)$, write $M^{\alpha}: \Delta\left(X^{H_{q}}\right)^{\text {op }} \rightarrow \underline{\mathrm{Ab}}$ for the functor obtained by restricting $M$; i.e., $M^{\alpha}$ sends an object $\sigma: \Delta^{m} \rightarrow X^{H_{q}}$ to $M(\bar{\sigma}(\alpha \times 1))$, where $\bar{\sigma}: G / H_{q} \times \Delta^{m} \rightarrow$ $X$ is the map adjoint to $\sigma$ and $\alpha \times 1: G / H_{0} \times \Delta^{m} \rightarrow G / H_{q} \times \Delta^{m}$ is the obvious map coming from $\alpha \in N_{q}(\mathscr{O}(G))$. Since $M$ factors through $v_{X}$, it follows that $M^{\alpha}$ factors through the canonical functor $\Delta\left(X^{H_{q}}\right) \rightarrow \Pi\left(X^{H_{q}}\right)$, i.e., $M^{\alpha}$ is a local system of coefficients on $X^{H_{q}}$. The spectral sequence for the bicomplex then takes the following form:

2.4. Lemma. There is a spectral sequence (natural in $X$ and $M$ )

$$
E_{1}^{p, q}=\prod_{\alpha \in N_{q}(\mathcal{O}(G))} H_{t w}^{p}\left(X^{\alpha(q)}, M^{\alpha}\right) \Rightarrow H_{G}^{p+q}(X, M)
$$

(where $\alpha(q)=H_{q}$ for $\alpha=\left(G / H_{0} \rightarrow \cdots \rightarrow G / H_{q}\right)$ and $H_{t w}^{p}$ is ordinary twisted cohomology).

The theorem now follows from this lemma and the nonequivariant case.

\section{The Serre SPECtral SeQuence}

Let $f: Y \rightarrow X$ be a $G$-fibration; i.e. (Bredon [1]), $f$ is a map of $G$-spaces that restricts to a Serre fibration $Y^{H} \rightarrow X^{H}$ for each subgroup $H \subseteq G$. Let $M: \Delta_{G}(Y)^{\text {op }} \rightarrow \underline{\mathrm{Ab}}$ be a local system of coefficients on $Y$. For $q \geq 0$ we define a functor

$$
H_{G}^{q}(f, M): \Delta_{G}(X)^{\mathrm{op}} \rightarrow \underline{\mathrm{Ab}}
$$

as follows: for an object $\left(\sigma: G / K \times \Delta^{n} \rightarrow X\right)$ of $\Delta_{G}(X)$, let $\sigma^{*}(Y)$ be the $G$-space obtained by pulling back $f$ along $\sigma$ :

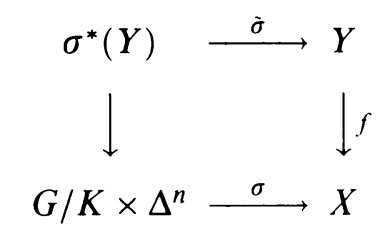


and define $H_{G}^{q}(f, M)(\sigma)=H_{G}^{q}\left(\sigma^{*}(Y), \tilde{\sigma}^{*} M\right)$. (This is clearly a functor of $\sigma$.) 3.1. Remarks. Note that $H_{G}^{q}(f, M)$ is a local system on $X$, i.e., $H_{G}^{q}(f, M)$ factors through the functor $v_{X}: \Delta_{G}(X) \rightarrow \pi_{G}(X)$. Indeed, if $u: \Delta^{m} \rightarrow \Delta^{n}$ is a simplicial map, then since $f$ is a $G$-fibration, the map $(\sigma(1 \times u))^{*}(Y) \rightarrow$ $\sigma^{*}(Y)$ covering $\sigma(1 \times u): G / K \times \Delta^{m} \rightarrow G / K \times \Delta^{n}$ is a weak $G$-homotopy equivalence. Hence it induces an isomorphism in Bredon cohomology with twisted coefficients, by Theorem 2.3. We shall denote the functor $\pi_{G}(X)^{\text {op }} \rightarrow$ Ab corresponding to $H_{G}^{q}(f, M)$ again by $H_{G}^{q}(f, M)$. This functor sends an object $(\tau: G / K \rightarrow X)$ of $\pi_{G}(X)$ to the group $H_{G}^{q}\left(\tau^{*}(Y), \tilde{\tau}^{*}(M)\right)$, where

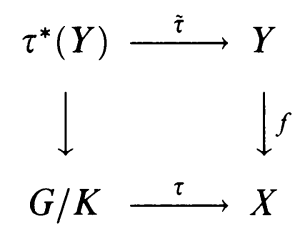

is a pullback of $G$-spaces. The $G$-space $\tau^{*}(Y)$ is the fiber over the "point" $\tau$ of $X$. The map $\tau$ corresponds to a point $t \in X^{K}$ by $\tau(g K)=g \cdot t$. The fiber $Y_{t}=f^{-1}(t)$ over $t$ is a $K$-space, and $G \times_{K} Y_{t} \cong \tau^{*}(Y)$ while $H_{G}^{q}\left(\tau^{*} Y, \tilde{\tau}^{*} M\right) \cong$ $H_{K}^{q}\left(Y_{t}, M \mid Y_{t}\right)$. In this sense, the functor $H_{G}^{*}(f, M): \pi_{G}(X)^{\mathrm{op}} \stackrel{\mathrm{Ab}}{\rightarrow}$ is Bredon cohomology of the fibers of $f$.

3.2. Theorem. For any G-fibration $f: Y \rightarrow X$, there is a natural spectral sequence

$$
E_{2}^{p, q}=H_{G}^{p}\left(X, H_{G}^{q}(f, M)\right) \Rightarrow H_{G}^{p+q}(Y, M) .
$$

In the proof of 3.2 , we shall use a simplicial version of 2.3 and 2.4 , which we briefly describe first. For a functor $X: \mathscr{O}(G)^{\text {op }} \rightarrow$ (ssets), let $\pi(X(-))$ be the functor that assigns to an object $G / H$ of $\mathscr{O}(G)$ the fundamental groupoid of the simplicial set $X(G / H)$. (This is the usual edge-path groupoid, which is isomorphic to the fundamental groupoid $\pi\left(|X(G / H)|, X_{0}(G / H)\right)$ of the realization of $X(G / H)$ with the vertices of $X(G / H)$ as base points.) Then let $\pi_{G}(X)=\int_{\mathscr{O}(G)} \pi(X(-))$. Moreover, write $\Delta_{G}(X)=\int_{\mathscr{O}(G) \times \Delta} X=\int_{\mathscr{O}(G)} \int_{\Delta} X$. Then as before, taking the "last vertex" yields a canonical functor $v_{X}: \Delta_{G}(X) \rightarrow$ $\pi_{G}(X)$. A local system of coefficients on $X$ is a functor $M: \Delta_{G}(X)^{\mathrm{op}} \rightarrow \underline{\mathrm{Ab}}$ that, up to isomorphism, factors through $v_{X}$. Completely analogous to 2.4 , there is a spectral sequence, for any such $M$,

$$
\prod_{u \in N_{q}(\mathscr{O}(G))} H_{t w}^{p}\left(X\left(G / H_{q}\right), M^{\alpha}\right) \Rightarrow H^{p+q}\left(\Delta_{G}(X), M\right) .
$$

It follows that a natural transformation $f: Y \rightarrow X$ between functors from $\mathscr{O}(G)^{\text {op }}$ into simplicial sets induces an isomorphism

$$
H^{n}\left(\Delta_{G}(X), M\right) \stackrel{\sim}{\rightarrow} H^{n}\left(\Delta_{G}(Y), f^{*} M\right),
$$

provided that for any subgroup $H$ of $G$ the map $f(G / H): Y(G / H) \rightarrow X(G / H)$ is a weak equivalence of simplicial sets.

Another preliminary remark for the proof of 3.2 concerns the operations of pulling back and of associating the singular simplicial sets $S_{\bullet}(U)$ to a topological space $U$. In general, these two operations do not commute (up to homotopy); but they do for fibrations. More precisely, let $f: V \rightarrow U$ be a map of 
topological spaces, let $\Delta(-, n) \in$ (ssets) be the representable simplicial set, and let $\Delta^{n}=|\Delta(-, n)| \in$ Top be the standard $n$-simplex, as before. For a map $\xi: \Delta^{n} \rightarrow U$, with corresponding simplicial map $\hat{\xi}: \Delta(-, n) \rightarrow S_{\bullet}(U)$, there is a simplicial comparison map

$$
\hat{\xi}^{*}\left(S_{\bullet}(V)\right) \rightarrow S_{\bullet}\left(\xi^{*}(V)\right)
$$

Here the left-hand side denotes the pullback of simplicial sets and the righthand side is the singular simplicial set associated to the pullback of spaces. The comparison map (34) is in general not a (weak) homotopy equivalence, but one easily checks that it is when $f$ is a fibration.

Proof of 3.2. The $G$-fibration $f: Y \rightarrow X$ induces a functor $\Delta_{G}(f): \Delta_{G}(Y) \rightarrow$ $\Delta_{G}(X)$, and we have a Grothendieck spectral sequence (cf. (10))

$$
H^{p}\left(\Delta_{G}(X), H^{q}\left(\Delta_{G}(f) /-, M\right)\right) \Rightarrow H^{p+q}\left(\Delta_{G}(Y), M\right) .
$$

It thus remains to be shown that the two functors $H^{q}\left(\Delta_{G}(f) /-, M\right)$ and $H_{G}^{q}(f, M)$ from $\Delta_{G}(X)^{\text {op }}$ into $\underline{\mathrm{Ab}}$ are isomorphic.

Fix an object $\left(\sigma: G / K \times \Delta^{n} \rightarrow X\right)$ of $\Delta_{G}(X)$. We shall construct an isomorphism $H^{q}\left(\Delta_{G}(f) / \sigma, M\right) \cong H_{G}^{q}(f, M)(\sigma)$, and it will be clear from the construction that this isomorphism is natural in $\sigma$. (Here and below, we denote also by $M$ any system of coefficients induced by $M$.) We first introduce some notation.

The map $\sigma: G / K \times \Delta^{n} \rightarrow X$ has an adjoint $\bar{\sigma}: \Delta^{n} \rightarrow X^{K}$ and a corresponding simplicial map $\hat{\sigma}: \Delta(-, n) \rightarrow S_{\bullet}\left(X^{K}\right)$ (as in (34)); we shall also write $\hat{\sigma}: \Delta(-, n) \rightarrow S_{\bullet}\left(X^{L}\right)$ for any subgroup $L \subseteq K$. Let $f^{\prime}: Y^{\prime}=f^{-1}\left(X^{K}\right) \rightarrow X^{K}$ be the fibration of $K$-spaces obtained by restricting $f$, and let $\bar{\sigma}^{*}\left(Y^{\prime}\right)$ be the $K$-space obtained by pulling back $f^{\prime}$ along $\bar{\sigma}$.

Observe that there is a canonical equivalence of categories

$$
\mathscr{O}(K) \rightarrow \mathscr{O}(G) /(G / K)
$$

that sends an object $K / L$ into the quotient-mapping $G / L \rightarrow G / K$.

We begin by analysing $H_{G}^{q}(f, M)(\sigma)$. There is a functor $(-\times 1)^{*} \sigma^{*}(Y)$ from $(\mathscr{O}(G) /(G / K))^{\text {op }}$ into spaces that send $\alpha: G / L \rightarrow G / K$ to the pullback of $f: Y \rightarrow X$ along $\sigma \circ(\alpha \times 1)$. One easily checks that there are canonical equivalences of categories

$$
\begin{aligned}
\Delta_{G}\left(\sigma^{*}(Y)\right) & \cong \int_{\mathscr{O}(G) /(G / K)} \Delta\left((-\times 1)^{*} \sigma^{*}(Y)\right) \\
& \cong \int_{\mathscr{O}(K)} \Delta\left(\bar{\sigma}^{*}\left(Y^{\prime}\right)^{(-)}\right) \cong \int_{\mathscr{O}(K)} \int_{\Delta} S_{\bullet}\left(\bar{\sigma}^{*}\left(Y^{\prime}\right)^{(-)}\right)
\end{aligned}
$$

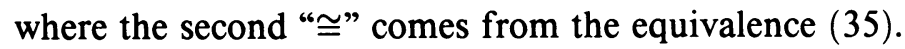

On the other hand, a direct computation shows that there is an equivalence of categories

$$
\Delta_{G}(f) / \sigma \cong \int_{\mathscr{O}(K)} \int_{\Delta} \hat{\sigma}^{*}\left(S_{\bullet}\left(Y^{(-)}\right)\right)
$$

Now since $f^{\prime}$ is a $K$-fibration, by $(34)$ there is a weak equivalence $\hat{\sigma}\left(S_{\bullet}\left(Y^{\prime L}\right)\right) \rightarrow$ $S_{\bullet}\left(\bar{\sigma}^{*}\left(Y^{\prime L}\right)\right)$ for any subgroup $L \subseteq K$, natural in $L$. Therefore we obtain an 
isomorphism of type (33), which in this particular case (by the equivalences (36) and (37)) takes the form

$$
H^{q}\left(\Delta_{G}\left(\sigma^{*}(Y)\right), M\right) \stackrel{\sim}{\rightarrow} H^{q}\left(\Delta_{G}(f) / \sigma, M\right) .
$$

This completes the proof.

\section{Multiplicative STRUCture}

With a little more care, it can be shown that the equivariant Serre spectral sequence (Theorem 3.2) carries a product structure, as expressed in the following result.

4.1. Theorem. For a G-fibration $f: Y \rightarrow X$ and local coefficients $M$ and $N$ on $Y$, there is a natural pairing of Serre spectral sequences

$$
E_{r}^{p, q}(M) \otimes E_{r}^{p^{\prime}, q^{\prime}}(N) \rightarrow E_{r}^{p+p^{\prime}, q+q^{\prime}}(M \otimes N)
$$

converging to the standard pairing

$$
H_{G}^{*}(Y, M) \otimes H_{G}^{*}(Y, N) \stackrel{\cup}{\rightarrow} H_{G}^{*}(Y, M \otimes N) .
$$

Furthermore, the pairing of $E_{2}$-terms agrees, up to the sign $(-1)^{p^{\prime} q}$, with the standard pairing

$$
H_{G}^{p}\left(X, H_{G}^{q}(f, M)\right) \otimes H_{G}^{p^{\prime}}\left(X, H_{G}^{q^{\prime}}(f, N)\right) \rightarrow H_{G}^{p+p^{\prime}}\left(X, H_{G}^{q+q^{\prime}}(f, M \otimes N)\right) .
$$

These "standard" pairings (38) and (39) are explicitly described in (51). Notice that for a local system $R$ of (commutative) rings on $Y$, the Serre spectral sequence becomes a spectral sequence of graded (commutative) rings, with $E_{2} \cong H_{G}^{*}\left(X, H_{G}^{*}(f, R)\right)$ as rings, modulo a sign.

The proof of Theorem 3.2 was based on a reduction to the Grothendieck spectral sequence (10), and Theorem 4.1 is a special case of the following product theorem for this Grothendieck spectral sequence:

4.2. Theorem. For any functor $f: \mathbb{D} \rightarrow \mathbb{C}$ and any coefficients $M, N \in \underline{\mathrm{Ab}}(\mathbb{D})$, there is a natural pairing of Grothendieck spectral sequences

$$
E_{r}(M) \otimes E_{r}(N) \rightarrow E_{r}(M \otimes N)
$$

converging to the standard pairing

$$
H^{*}(\mathbb{D}, M) \otimes H^{*}(\mathbb{D}, N) \rightarrow H^{*}(\mathbb{D}, M \otimes N) .
$$

Furthermore, the pairing of $E_{2}$-terms agrees up to the sign $(-1)^{p^{\prime} q}$ with the standard pairing

$$
H^{p}\left(\mathbb{C}, H^{q}(f, M)\right) \otimes H^{p^{\prime}}\left(\mathbb{C}, H^{q^{\prime}}(f, N)\right) \rightarrow H^{p+p^{\prime}}\left(\mathbb{C}, H^{q+q^{\prime}}(f, M \otimes N)\right) .
$$

Although various cases of this result are well known, it seems worthwhile to prove it in the stated generality. To this end, recall first that for a small category $\mathbb{C}$, the category of contravariant abelian group-valued functors is denoted by $\underline{A b}(\mathbb{C})$. In this category tensor-products are formed "pointwise"; i.e., $M \otimes M^{\prime}(C)=M(C) \otimes_{\mathbb{Z}} M^{\prime}(C)$ for any $M, M^{\prime} \in \underline{\mathrm{Ab}}(\mathbb{C})$ and any $C \in \mathbb{C}$. For a functor $S: \mathbb{C}^{\text {op }} \rightarrow$ Sets, $\mathbb{Z}[S]$ denotes the object of $\underline{\mathrm{Ab}}(\mathbb{C})$ obtained by composing $S$ with the free abelian group functor. In particular, for each representable functor $\mathbb{C}(-, C)$ one has a "representable" object $\mathbb{Z}[\mathbb{C}(-, C)]$ of 
$\underline{\mathrm{Ab}}(\mathbb{C})$, characterized by the property that the action of $\mathbb{C}$ on $M$ induces a natural isomorphism

$$
\operatorname{Hom}(\mathbb{Z}[\mathbb{C}(-, C)], M) \stackrel{\sim}{\rightarrow} M(C),
$$

for each $M \in \underline{\mathrm{Ab}}(\mathbb{C})$. It follows that $\mathbb{Z}[\mathbb{C}(-, C)]$ is a projective object. More generally, if $P \in \underline{\mathrm{Ab}}(\mathbb{C})$ is projective, then the canonical surjection $\sum_{C \in \mathbb{C}, x \in P(C)} \mathbb{Z}[\mathbb{C}(-, C)] \rightarrow P \rightarrow 0$ must split. So $P$ is a retract of a sum of representables. Thus $P$ is pointwise free, and hence flat (w.r.t. the pointwise tensor product).

We shall also use a different tensor product on abelian functors. If $M \in$ $\underline{\mathrm{Ab}}(\mathbb{C})$ and $N \in \underline{\mathrm{Ab}}\left(\mathbb{C}^{\text {op }}\right)$, one can form an abelian group

$$
M \otimes_{\mathbb{C}} N,
$$

by first taking the sum $\sum_{C \in \mathbb{C}} M(C) \otimes_{\mathbb{Z}} N(C)$, and then factoring out the relations $x \otimes N(\alpha)(y)=M(\alpha)(x) \otimes y$, for any arrow $\alpha: C \rightarrow C^{\prime}$ in $\mathbb{C}$ and any $x \in M\left(C^{\prime}\right), y \in M(C)$. More generally, for $M \in \underline{\mathrm{Ab}}(\mathbb{C})$ and $N \in \underline{\mathrm{Ab}}\left(\mathbb{D} \times \mathbb{C}^{\text {op }}\right)$, one obtains a tensor product $M \otimes_{\mathbb{C}} N \in \underline{\mathrm{Ab}}(\mathbb{D})$ by applying this construction (41) pointwise: for any object $D \in \mathbb{D}$,

$$
\left(M \otimes_{\mathbb{C}} N\right)(D)=M \otimes_{\mathbb{C}} N(D,-) .
$$

This tensor product satisfies the usual adjunction formulas

$$
\begin{gathered}
\operatorname{Hom}_{\mathbb{D}}\left(M \otimes_{\mathbb{C}} N, L\right) \cong \operatorname{Hom}_{\mathbb{C}}\left(M, \underline{\operatorname{Hom}}_{\mathbb{D}}(N, L)\right), \\
\operatorname{Hom}_{\mathbb{D}}\left(M \otimes_{\mathbb{C}} N, L\right) \cong \operatorname{Hom}_{\mathbb{D} \otimes \mathbb{C o p}}(N, \underline{\operatorname{Hom}}(M, L)),
\end{gathered}
$$

where, on the right, $\underline{\operatorname{Hom}}_{\mathbb{D}}(N, L) \in \underline{\mathrm{Ab}}(\mathbb{C})$ is defined by having value $\operatorname{Hom}_{\mathbb{D}}(N(-, C), L)$ at any object $C \in \mathbb{C}$, while $\underline{\operatorname{Hom}}(M, L) \in \underline{\mathrm{Ab}}\left(\mathbb{D} \times \mathbb{C}^{\text {op }}\right)$ has value $\operatorname{Hom}(M(C), L(D))$ at $(D, C)$. Several standard facts follow purely formally from these adjunction formulas. For example, by (40) one obtains isomorphisms

$$
\begin{aligned}
& \mathbb{Z}[\mathbb{C}(-, C)] \otimes_{\mathbb{C}} N \cong N(-, C), \\
& M \otimes_{\mathbb{C}} \mathbb{Z}[\mathbb{C}(C,-) \times \mathbb{D}(-, D)] \cong M(C) \otimes \mathbb{Z}[\mathbb{D}(-, D)] .
\end{aligned}
$$

Also, purely formally, (44) and (45) readily yield that if $N$ is projective in $\underline{\mathrm{Ab}}\left(\mathbb{D} \times \mathbb{C}^{\text {op }}\right)$ then so is $N(-, C) \in \mathrm{Ab}(\mathbb{D})$, for each $C \in \mathbb{C}$. It then follows by (43) that if $M$ and $N$ are projective so is $M \otimes_{\mathbb{C}} N$. Notice also that since every projective object (in $\underline{A b}(\mathbb{C})$ or in $\underline{A b}\left(\mathbb{D} \times \mathbb{C}^{\text {op }}\right)$ ) is a retract of a sum of representables, formulas (45) and (46) yield that if $M$ and $N$ are projective then the functors $M \otimes_{\mathbb{C}}(-)$ and $(-) \otimes_{\mathbb{C}} N$ are exact. In brief, projectives are flat with respect to the tensor product (42). Finally, we remark that the tensor product (42) distributes over the pointwise tensor product, in the weak sense that there is an evident map

$$
\psi:\left(M \otimes M^{\prime}\right) \otimes_{\mathbb{C}}\left(N \otimes N^{\prime}\right) \rightarrow\left(M \otimes_{\mathbb{C}} N\right) \otimes\left(M^{\prime} \otimes_{\mathbb{C}} N^{\prime}\right)
$$

that is compatible with the adjunction isomorphisms (45) and (46). (However, in general $\psi$ is not an isomorphism.)

Next, we consider pairings for the cohomology of categories. For a small category $\mathbb{C}$ and for $M \in \underline{\mathrm{Ab}}(\mathbb{C})$, recall the cohomology groups $H^{n}(\mathbb{C}, M)$ from (2). These can also be constructed as ext-groups $H^{n}(\mathbb{C}, M) \cong \operatorname{Ext}_{\underline{\mathrm{Ab}}(\mathbb{C})}^{n}\left(K_{\mathbb{Z}}, M\right)$ 
where $K_{\mathbb{Z}} \in \underline{\mathrm{Ab}}(\mathbb{C})$ is the constant functor with value $\mathbb{Z}$, as is clear from the isomorphism $\operatorname{Hom}_{\mathbb{C}}\left(K_{\mathbb{Z}}, M\right) \cong \Gamma M=\lim M$.

Now consider for $A \in \underline{\mathrm{Ab}}(\mathbb{C})$ a projective resolution $P_{\bullet} \rightarrow A \rightarrow 0$. Since projectives are flat and since the tensor product of projectives is again projective, it follows that if Tor $^{\mathbb{Z}}(A(C), A(C))=0$ for all objects $C \in \mathbb{C}$ then $P_{\bullet} \otimes P_{\bullet} \rightarrow$ $A \otimes A \rightarrow 0$ is again a projective resolution. Now suppose, in addition, that we are given a coproduct $\Delta_{A}: A \rightarrow A \otimes A$ on $A$. By standard homological algebra, this map $\Delta_{A}$ can be lifted to a map of chain complexes

$$
\Delta_{P_{\bullet}}: P_{\bullet} \rightarrow P_{\bullet} \otimes P_{\bullet},
$$

unique up to chain homotopy equivalence. Furthermore, by this uniqueness, if $\Delta_{A}$ is commutative (coassociative) then $\Delta_{P_{0}}$ is graded commutative (coassociative) up to chain homotopy. The lifting (48) yields for any $M, N \in \underline{\mathrm{Ab}}(\mathbb{C})$ a pairing

$$
\operatorname{Ext}_{\underline{\mathrm{Ab}}(\mathbb{C})}^{*}(A, M) \otimes \operatorname{Ext}_{\underline{\mathrm{Ab}}(\mathbb{C})}^{*}(A, N) \rightarrow \operatorname{Ext}_{\underline{\mathrm{Ab}}(\mathbb{C})}^{*}(A, M \otimes N),
$$

by composing the tensor product map

$$
\operatorname{Hom}\left(P_{\bullet}, M\right) \otimes \operatorname{Hom}\left(P_{\bullet}, N\right) \rightarrow \operatorname{Hom}\left(P_{\bullet} \otimes P_{\bullet}, M \otimes N\right)
$$

with the map

$$
\operatorname{Hom}\left(P_{\bullet} \otimes P_{\bullet}, M \otimes N\right) \rightarrow \operatorname{Hom}\left(P_{\bullet}, M \otimes N\right)
$$

induced by $\Delta_{P_{0}}$. In the appropriate sense, this graded pairing (49) is commutative (and associative) whenever the map $\Delta_{A}: A \rightarrow A \otimes A$ is. For the special case where $A=K_{\mathbb{Z}}$ and $\Delta_{A}$ is the natural isomorphism $K_{\mathbb{Z}} \stackrel{\sim}{\rightarrow} K_{\mathbb{Z}} \otimes K_{\mathbb{Z}}$, this pairing gives a natural graded commutative and associative pairing

$$
H^{*}(\mathbb{C}, M) \otimes H^{*}(\mathbb{C}, N) \rightarrow H^{*}(\mathbb{C}, M \otimes N) .
$$

This pairing is independent of the choice of the resolution $P_{\bullet}$ and the lifting $\Delta_{P_{0}}$; a fact that will be of importance below. For a $G$-space $X$ and for $\mathbb{C}=$ $\Delta_{G}(X)$ this gives a pairing

$$
\cup: H_{G}^{*}(X, M) \otimes H_{G}^{*}(X, N) \rightarrow H_{G}^{*}(X, M \otimes N)
$$

for local coefficients $M$ and $N$. (Recall from (19) that we only use the notation $H_{G}^{*}(X, M)$ if $M$ is local; also observe that for a local system $R$ of rings, the multiplication $R \otimes R \rightarrow R$ and the pairing (51) together give a ring-structure on $H_{G}^{*}(X, R)$.)

After these preparations, we return to 4.2 .

Proof of Theorem 4.2. Suppose given a functor $f: \mathbb{D} \rightarrow \mathbb{C}$ and coefficient systems $M, N$ in $\underline{\mathrm{Ab}}(\mathbb{D})$ as in the statement of the theorem. Let $P_{\bullet} \rightarrow K_{\mathbb{Z}} \rightarrow$ 0 and $Q_{\bullet} \rightarrow \mathbb{Z}[\mathbb{C}(f(-), ?)] \rightarrow 0$ be projective resolutions in $\underline{\mathrm{Ab}}(\mathbb{C})$ and $\underline{\mathrm{Ab}}\left(\mathbb{D} \times \mathbb{C}^{\text {op }}\right)$, respectively. As in (48), one can lift the coproduct $K_{\mathbb{Z}} \stackrel{\sim}{\rightarrow} K_{\mathbb{Z}} \otimes K_{\mathbb{Z}}$ and the diagonal $\mathbb{Z}[\mathbb{C}(f(-), ?)] \rightarrow \mathbb{Z}[\mathbb{C}(f(-), ?)] \otimes \mathbb{Z}[\mathbb{C}(f(-)$, ?)] to coproducts $\Delta_{P_{\bullet}}: P_{\bullet} \rightarrow P_{\bullet} \otimes P_{\bullet}$ and $\Delta_{Q_{\bullet}}: Q_{\bullet} \rightarrow Q_{\bullet} \otimes Q_{\bullet}$. Next, by taking tensor products over $\mathbb{C}$, we obtain a projective resolution in $\underline{A b}(\mathbb{D})$,

$$
P_{\bullet} \otimes_{\mathbb{C}} Q_{\bullet} \rightarrow K_{\mathbb{Z}} \otimes_{\mathbb{C}} \mathbb{Z}[\mathbb{C}(f(-), ?)] \cong K_{\mathbb{Z}} \rightarrow 0 .
$$

Indeed, we have observed that the $\otimes_{\mathbb{C}}$-tensor product of projectives is projective and that these projectives are flat (w.r.t. $\otimes_{\mathbb{C}}$ ). Furthermore, for fixed $D \in$ 
$\mathbb{D}$ the object $\mathbb{Z}[\mathbb{C}(f(-)$, ?)] becomes the representable $\mathbb{Z}[\mathbb{C}(f(D)$, ? $)]$, so that $\mathbb{Z}\left[\mathbb{C}\left(f(-)\right.\right.$, ?] is also flat for $\otimes_{\mathbb{C}}$; the isomorphism in (52) follows by applying (42) and then (46). For this resolution (52), we now construct a particular lifting

$$
\tilde{\Delta}: P_{\bullet} \otimes_{\mathbb{C}} Q_{\bullet} \rightarrow\left(P_{\bullet} \otimes_{\mathbb{C}} Q_{\bullet}\right) \otimes\left(P_{\bullet} \otimes_{\mathbb{C}} Q_{\bullet}\right)
$$

of the canonical coproduct $K_{\mathbb{Z}} \stackrel{\sim}{\rightarrow} K_{\mathbb{Z}} \otimes K_{\mathbb{Z}}$ in $\underline{\mathrm{Ab}}(\mathbb{D})$. First, the liftings $\Delta_{P_{\mathbf{0}}}$ and $\Delta_{Q_{\bullet}}$ together give a map $\Delta_{P_{\bullet}} \otimes \Delta_{Q_{\bullet}}: P_{\bullet} \otimes_{\mathbb{C}} \overline{Q_{\bullet}} \rightarrow\left(P_{\bullet} \otimes P_{\bullet}\right) \otimes_{\mathbb{C}}\left(Q_{\bullet} \otimes Q_{\bullet}\right)$. Furthermore, the natural map $\psi:\left(P_{p} \otimes P_{p^{\prime}}\right) \otimes_{\mathbb{C}}\left(Q_{q} \otimes Q_{q^{\prime}}\right) \rightarrow\left(P_{p} \otimes_{\mathbb{C}} Q_{q}\right) \otimes$ $\left(P_{p^{\prime}} \otimes \mathbb{C} Q_{q^{\prime}}\right)$ given by $(47)$ becomes a morphism $\psi^{\prime}$ of complexes when modified as $\psi^{\prime}=(-1)^{p^{\prime} q} \psi$. The desired map (53) of complexes is now defined as $\widetilde{\Delta}=\psi^{\prime} \circ\left(\Delta_{P_{\bullet}} \otimes \Delta_{Q_{\bullet}}\right)$.

As before, this lifting (53) yields for any $M$ and $N$ in $\underline{\mathrm{Ab}}(\mathbb{D})$ a pairing

$\operatorname{Hom}_{\mathbb{D}}\left(P_{\bullet} \otimes_{\mathbb{C}} Q_{\bullet}, M\right) \otimes \operatorname{Hom}_{\mathbb{D}}\left(P_{\bullet} \otimes_{\mathbb{C}} Q_{\bullet}, N\right) \rightarrow \operatorname{Hom}_{\mathbb{D}}\left(P_{\bullet} \otimes_{\mathbb{C}} Q_{\bullet}, M \otimes N\right)$

that (as any lifting of $K_{\mathbb{Z}} \stackrel{\sim}{\rightarrow} K_{\mathbb{Z}} \otimes K_{\mathbb{Z}}$ ) induces the usual pairing

$$
H^{*}(\mathbb{D}, M) \otimes H^{*}(\mathbb{D}, N) \rightarrow H^{*}(\mathbb{D}, M \otimes N) .
$$

We complete the proof of the theorem by inspecting the spectral sequence given by the double complex $\operatorname{Hom}_{\mathbb{D}}\left(P_{\bullet} \otimes_{\mathbb{C}} Q_{\bullet}, M\right)$. The "coproducts" $\Delta_{P_{\bullet}}$ and $\Delta_{Q_{\bullet}}$ equip this double complex and hence this spectral sequence with a pairing

$$
E_{r}^{p, q}(M) \otimes E_{r}^{p^{\prime}, q^{\prime}}(N) \rightarrow E_{r}^{p+p^{\prime}, q+q^{\prime}}(M \otimes N) .
$$

From the adjunction formula

$$
\operatorname{Hom}_{\mathbb{D}}\left(P_{p} \otimes_{\mathbb{C}} Q_{q}, M\right) \cong \operatorname{Hom}_{\mathbb{C}}\left(P_{p}, \underline{\operatorname{Hom}}_{\mathbb{D}}\left(Q_{q}, M\right)\right)
$$

and the fact that $Q_{\bullet} \rightarrow \mathbb{Z}[\mathbb{C}(f(-)$, ?)] $\rightarrow 0$ is a resolution, we find that this spectral sequence takes the form

$$
E_{2}^{p, q}(M) \cong H^{p}\left(\mathbb{C}, \mathscr{E} x t^{q}(\mathbb{Z}[\mathbb{C}(f(-), ?)], M)\right) \Rightarrow H^{p+q}(\mathbb{D}, M),
$$

where $\mathscr{E} x t^{q} \in \mathrm{Ab}(\mathbb{C})$ is the functor $C \mapsto \operatorname{Ext}_{\mathrm{Ab}(\mathbb{D})}^{q}(\mathbb{Z}[\mathbb{C}(f(-), C)], M)$; all this is natural in $M$. Furthermore, since the adjunction formula $(55)=(43)$ is compatible with the map $\psi$ of (47), the isomorphism in (56) respects pairings, up to sign (due to modification of $\psi$ ). The proof is now complete by the observation that (56) is essentially the standard Grothendieck spectral sequence (10). Indeed, for a fixed object $C \in \mathbb{C}$ there is an evident isomorphism of categories

$$
\int_{\mathbb{D}} \mathbb{C}(f(-), C) \cong f / C,
$$

natural in $C$. By the following lemma, the functor $\mathscr{E} x t^{q}(\mathbb{Z}[\mathbb{C}(f(-), ?)], M)$ occurring in (56) is then naturally isomorphic to the functor $H^{n}(f /(-), M)$, by an isomorphism compatible with the pairings.

Before we state the lemma, we recall that for any small category $\mathbb{B}$ and any functor $X: \mathbb{B}^{\text {op }} \rightarrow$ Sets, $\int_{\mathbb{B}} X$ is the category with as objects the pairs $(B, x)$ where $x \in X(B)$ and as arrows $\alpha:(B, x) \rightarrow\left(B^{\prime}, x^{\prime}\right)$ arrows $\alpha: B \rightarrow B^{\prime}$ with $X(\alpha)\left(x^{\prime}\right)=x$. There is an evident projection functor $\pi: \int_{\mathbb{B}} X \rightarrow \mathbb{B}$. 
Lemma. For any small category $\mathbb{B}$ and any functors $X: \mathbb{B}^{\text {op }} \rightarrow$ Sets and $M: \mathbb{B}^{\text {op }}$ $\rightarrow \underline{\mathrm{Ab}}$, there is a canonical isomorphism

$$
\operatorname{Ext}_{\underline{\mathrm{Ab}(\mathbb{B})}}^{q}(\mathbb{Z}[X], M) \cong H^{n}\left(\int_{\mathbb{B}} X, \pi^{*} M\right) \quad(q \geq 0)
$$

compatible with pairings.

Proof. The functor $\pi^{*}: \underline{\mathrm{Ab}}(\mathbb{B}) \rightarrow \underline{\mathrm{Ab}}\left(\int_{\mathbb{B}} X\right)$ given by composition with $\pi$ is exact and has an exact left adjoint $\pi_{!}: \underline{\mathrm{Ab}}\left(\int_{\mathbb{B}} X\right) \rightarrow \underline{\mathrm{Ab}}(\mathbb{B})$, given for $N \in$ $\underline{\mathrm{Ab}}\left(\int_{\mathbb{B}} X\right)$ and $B \in \mathbb{B}$ by

$$
\pi_{!}(N)(B)=\sum_{x \in X(B)} N(x, B) .
$$

By the adjunction formula $\operatorname{Hom}\left(\pi_{!} N, M\right) \cong \operatorname{Hom}\left(N, \pi^{*} M\right), \pi_{!}$preserves projectives since $\pi^{*}$ is right exact. Thus if $P_{\bullet} \rightarrow K_{\mathbb{Z}} \rightarrow 0$ is a projective resolution in $\underline{\mathrm{Ab}}\left(\int_{\mathbb{B}} X\right)$, the complex $\operatorname{Hom}\left(P_{\bullet}, \pi^{*} M\right)$, to compute the righthand side of $(57)$, is isomorphic to the complex $\operatorname{Hom}\left(\pi_{!}\left(P_{\bullet}\right), M\right)$, to compute $\operatorname{Ext}^{\bullet}\left(\pi_{!}\left(K_{\mathbb{Z}}\right), M\right)$. The isomorphism (57) now follows by the observation that $\pi_{!}\left(K_{\mathbb{Z}}\right)=\mathbb{Z}[X]$. Next we show that this isomorphism is compatible with the usual pairing on the right of (57) (induced by the isomorphism $K_{\mathbb{Z}} \stackrel{\sim}{\rightarrow} K_{\mathbb{Z}} \otimes K_{\mathbb{Z}}$ in $\left.\underline{\mathrm{Ab}}\left(\int_{\mathbb{Z}} X\right)\right)$ and the pairing on the left of (57) induced by the diagonal $\mathbb{Z}[X] \rightarrow \mathbb{Z}[X] \otimes \mathbb{Z}[X]=\mathbb{Z}[X \times X]$. First note that a lifting $\Delta=\Delta_{P_{0}}: P_{\bullet} \rightarrow$ $P_{\bullet} \otimes P_{\bullet}$ of $K_{\mathbb{Z}} \stackrel{\sim}{\rightarrow} K_{\mathbb{Z}} \otimes K_{\mathbb{Z}}$ above induces a lifting $\Delta^{\prime}=\delta \circ \pi_{!}\left(\Delta_{P_{\bullet}}\right): \pi_{!}\left(P_{\bullet}\right) \rightarrow$ $\pi_{!}\left(P_{\bullet} \otimes P_{\bullet}\right) \rightarrow \pi_{!}\left(P_{\bullet}\right) \otimes \pi_{!}\left(P_{\bullet}\right)$, where $\delta$ is the evident "diagonal" map. The required compatibility now follows from the observation that under the adjunction between $\pi_{!}$and $\pi^{*}$, the map $\operatorname{Hom}\left(P_{\bullet}, \pi^{*} M\right) \otimes \operatorname{Hom}\left(P_{\bullet}, \pi^{*} N\right) \rightarrow$ $\operatorname{Hom}\left(P_{\bullet}, \pi^{*}(M) \otimes \pi^{*}(N)\right)=\operatorname{Hom}\left(P_{\bullet}, \pi^{*}(M \otimes N)\right)$ induced by $\Delta$ corresponds to the map $\operatorname{Hom}\left(\pi_{!}\left(P_{\bullet}\right), M\right) \otimes \operatorname{Hom}\left(\pi_{!}\left(P_{\bullet}\right), N\right) \rightarrow \operatorname{Hom}\left(\pi_{!}\left(P_{\bullet}\right), M \otimes N\right)$ induced by $\Delta^{\prime}$.

This proves the lemma, and thus completes the proof of Theorem 4.2.

\section{ACKNOWLEDGMENTS}

A first draft of this paper was written while both authors were visiting the mathematics department of the University of Chicago. We are grateful to this department for its hospitality and to Peter May for all he taught us about equivariant topology. We also thank the referee for his pertinent comments.

\section{REFERENCES}

1. G. E. Bredon, Equivariant cohomology theories, Lecture Notes in Math., vol. 34, Springer, Berlin and New York, 1967.

2. T. Bröcker, Singuläre definition der äquivarianten Bredon-Homologie, Manuscripta Math. 5 (1971), 91-102.

3. A. Dold and D. Puppe, Homologie nicht-additiver Funktoren. Anwendungen, Ann. Inst. Fourier (Grenoble) 11 (1961), 202-312.

4. A. D. Elmendorf, Systems of fixed point sets, Trans. Amer. Math. Soc. 277 (1983), 275-284.

5. P. Gabriel and M. Zisman, Calculus of fractions and homotopy theory, Ergeb. Math. Grenzgeb. (3), vol. 35, Springer, Berlin, 1966.

6. J. W. Gray, Fibred and cofibred categories (Proc. Conf. Categorial Algebra, La Jolla), Springer, Berlin and New York, 1965, pp. 21-83. 
7. A. Grothendieck, Sur quelques points d'algèbre homologique, Tôhoku Math. J. 9 (1957), 119-221.

8. I. Moerdijk and J.-A. Svensson, A Shapiro lemma for diagrams of spaces, with applications to equivariant topology (to appear).

9. D. Quillen, Higher algebraic K-theory. I, Lecture Notes in Math., vol. 341, Springer, Berlin and New York, 1973, pp. 85-147.

10. G. Segal, Categories and cohomology theories, Topology 13 (1974), 293-312.

11. R. M. Seymour, Some functorial constructions on G-spaces, Bull. London Math. Soc. 15 (1983), 353-359.

12. R. W. Thomason, Homotopy colimits in the category of small categories, Math. Proc. Cambridge Philos. Soc. 85 (1979), 91-109.

13. C. E. Watts, A homology theory for small categories (Proc. Conf. Categorical Algebra, La Jolla 1965), Springer, Berlin and New York, 1966, pp. 331-335.

Department of Mathematics, Rijksuniversiteit te Utrecht, Budapestlaan 6, P. O. Box 80.010, 3508 TA Utrecht, The Netherlands

E-mail address: moerdijk@math.run.nl

Department of Mathematics, Chalmers University, S-41296 Göteborg, Sweden

E-mail address: svensson@math.chalmers.se 